\title{
Estudio del Talento en Alumnos de Educación Infantil y Primaria
}

\author{
Ma Pilar Martín Lobo
}

Instituto de Neuropsicología y Educación,

Universidad Complutense de Madrid, C.U. Villanueva

\section{España}




\section{Resumen}

Introducción. La identificación del talento es el primer paso del proceso que se lleva a cabo para dar respuesta educativa a los alumnos con talento y altas capacidades. ¿Podemos identificar el talento desde edades tempranas? ¿El talento permanece de forma natural a través de las diferentes edades escolares o, por el contrario, es necesaria su orientación para que se desarrolle? El estudio de la identificación del talento es uno de los temas en los que más se está investigando y en el que se están aportando nuevos instrumentos, de gran interés y fáciles de aplicar. En este artículo se presenta el estudio realizado con una amplia muestra para identificar el talento musical desde Educación Infantil hasta $6^{\circ}$ de Primaria.

Método. El estudio se centra en la identificación de un tipo de talento: el talento musical. El método empleado en este estudio consistió en la aplicación de un Cuestionario a alumnos de Educación Infantil y Primaria, por parte de los profesores de Música. El Cuestionario fue seleccionado y adaptado del de Inteligencias Múltiples (Gardner, 1998). Se registraron las respuestas del Cuestionario seleccionado y, a continuación, se analizaron e interpretaron los resultados y se elaboraron las conclusiones.

Resultados. Los datos obtenidos reflejan que en Educación Infantil se da un porcentaje de alumnos con talento musical, pero esta cifra va disminuyendo con el paso del tiempo, de forma que se observa que en $6^{\circ}$ de Primaria no muestran talento musical mas que algunos alumnos de la amplia muestra estudiada.

Discusión. Concluimos que: i) Ya desde Educación Infantil es posible identificar el talento musical, como afirman diferentes autores. ii) A partir de Primaria se observa una disminución de alumnos con talento musical. iii) Para que el talento se desarrolle y permanezca en las sucesivos cursos de Primaria, se requiere su orientación y desarrollo.

Palabras Clave / Keywords: Talento, superdotación, altas capacidades, identificación e Inteligencias Múltiples. 


\section{Introducción}

Ante la necesidad de reconocer y orientar a los alumnos con altas capacidades, superdotados o con talento, los profesionales de la psicología y la psicopedagogía se encuentran con varias dificultades que podríamos agrupar en dos de las consideradas como mas importantes: cómo identificar el talento y la superdotación y cómo intervenir para desarrollar el talento y la superdotación identificados en el entorno escolar y familiar. Diferentes estudios e investigaciones publicadas explican modelos, pruebas de identificación, programas de intervención y procedimientos de orientación para profesores y padres (entre los numerosos estudios y experiencias podemos destacar los de R. Sternberg, 1995; J. Renzulli, 1977 - 1994; F. J. Monks, 1994; Y. Benito, 1996 y 1999; D. Prieto, 1996; J. Tourón, F. Peralta y R. Reparaz, 1998; E. Sánchez Manzano, 1999 y 2000; L. Pérez, P. Dominguez y E. Alfaro, 2000; P. Martín Lobo, 2004).

Respecto al primero de los problemas, consistente en cómo identificar el talento y la superdotación, conviene aclarar lo que entendemos por estos términos. A partir del paradigma cognitivo, la superdotación se entiende como una elevada capacidad intelectual que se manifiesta en todos y cada uno de los recursos intelectuales y se pueden medir mediante las puntuaciones altas en un conjunto variado de test (un centil 75 resulta una operativización razonable, A. Castelló y C. Batlle, 1996). Los talentosos, en cambio, muestran una aptitud elevada en alguno de los diferentes tipos de procesamiento evaluados, por lo que hace referencia a la especifidad, no a la generalidad de las aptitudes (J.Birch, 1984 y A. Gómez R. I. Rodriguez, 1993).

La teoría de las Inteligencias Múltiples ha llevado a considerar diferentes tipos de inteligencia y de talentos relacionados con cada una de ellas, las cuales requieren orientación y los medios adecuados para su desarrollo (Gardner, 1983, 1986). Uno de estos talentos es el musical que nos ha parecido idóneo para nuestra investigación y, en consecuencia, es el seleccionado para nuestro estudio.

La inteligencia musical no ha sido considerada como una habilidad en la cultura occidental, sino como un talento, destreza o pericia. Los niños tienen una capacidad natural para la música antes de comenzar los años escolares, pero, muchas veces, los centros educativos y los padres no dan toda la importancia que tiene el desarrollo musical (Gardner, 1993, 1995). 
La identificación del talento musical se centra en las habilidades para apreciar, discriminar, transformar y expresar las formas musicales, así como para ser sensible al ritmo, el tono y el timbre. Los instrumentos de identificación del talento se han centrado en cuestionarios, como el elaborado por Gardner, y en torno a los que se han ido diseñando modelos de identificación de las altas habilidades y del talento (H.Gardner, 1983; A. Castelló y C. Batlle, 1996).

El objetivo del presente estudio fue investigar el número de sujetos que mostraban talento musical a los 5 años, desde Educación Infantil, y a los 6, 7, 8, 9, 10 y 11 años, en la etapa que consideramos de Educación Primaria, con el fin de conocer si el talento musical permanece en el mismo número de sujetos a lo largo de los años de esta etapa escolar.

El modelo teórico que orienta la investigación se centra en la Teoría de las Inteligencias Múltiples, en la evaluación de las habilidades específicas referidas a las propias del talento musical (H. Gardner, D. Feldman y M. Krechevsky, 1998a), mediante la observación estructurada; además, se ha tomado como referencia la distinción entre los distintos tipos de valoración del talento simple, el talento complejo y las altas habilidades o superdotación (A. Castelló y C. Batlle, 1998). El talento musical, en este sentido, ha sido valorado para su identificación con un Centil de 90 o superior en el aspecto musical (referencia para identificación de talentos de J. S. Renzulli, 1977 entre otras).

En la relación de capacidades clave del Proyecto Spectrum, se consideran las siguientes como propias del talento musical, según adaptación de J. O. Chen (Ed) (1998): Project Spectrum: Early Learning Activities:

- Percepción:

- Es sensible a la dinámica (fuerte y suave).

- Es sensible al compás y a las pautas rítmicas.

- Discrimina el tono.

- Identifica los estilos musicales y los músicos.

- Reconoce los instrumentos y los sonidos diferentes. 
- Producción:

- Es capaz de mantener el tono preciso.

- Puede mantener el compás y las pautas rítmicas precisas.

- Manifiesta expresividad cuando canta o toca un instrumento.

- Puede recorda y reproducir las propiedades musicales de los sonidos y las otras composiciones.

- Composición:

- Realiza composiciones sencillas con ciertos caracteres propios del comienzo, el medio y el final.

- Crea un sistema sencillo de notación.

Por otra parte, las observaciones que los profesores hacen de los alumnos, mientras realizan las actividades, son claves y constituyen la herramienta básica para las valoraciones (El Proyecto Spectrum: Tomo l. Como construir sobre las capacidades infantiles, págna 145, Gardner y otros, 2000). Por esta razón, el profesor de música de los centros escolares donde se realizó el estudio, ha participado activamente en el estudio realizado.

\section{Método}

El método que se llevó a cabo consistió en que los profesores de música de los sujetos de la muestra valoraron cada uno de los items con 0,1 ó 2 puntos, del cuestionario seleccionado de cada uno de los sujetos. Para ello, llevaron a cabo un plan de observación estructurada en las actividades musicales, en el ámbito natural, de forma contextualizada (Th. Armstrong 1994, 1999). Se analizaron todos los resultados.

\section{Sujetos.}

La muestra está formada por de Educación Infantil y de Primaria, de los cuales se seleccionó una muestra de 485 niños y niñas de 5 a 11 años de edad. La muestra inicial era de 550, pero en uno de los colegios se trabajaba el Area Artística en cuanto al programa de Expresión Plástica, pero no se trabajaba el Programa de Música, por lo que se rechazó esta parte de la muestra (65 alumnos). Había un amplio espectro de estatus social y económico entre los familiares de los participantes en el estudio. 


\section{Materiales y procedimientos}

Se aplicó un Cuestionario para Identificación de Inteligencias Múltiples, apartado de Inteligencia Musical, anteriormente citado, y adaptado por M. D. Prieto (1997). Además, los propios alumnos de $2^{\circ}, 3^{\circ}, 4^{\circ}, 5^{\circ}$ y $6^{\circ}$ de Primaria participaron en el proceso y contestaron a unas preguntas que reflejaban conductas propias del talento musical y observaciones relacionadas con la posible realización de actividades musicales en el ámbito familiar, escolar y extraescolar.

En primer lugar, se planteó el estudio en el contexto de la actividad docente universitaria de la asignatura de Psicología de la Excepcionalidad, perteneciente a los estudios de Psicopedagogía: Se estudiaron los conceptos de superdotación, talento y altas capacidades, los instrumentos y pruebas de identificación y los programas de intervención. Se planteó a todos los alumnos de Psicopedagogía la oportunidad de realizar el estudio de Identificación de un tipo de talento y ocho alumnos decidieron libremente participar en el estudio. Se organizó un grupo de trabajo y se planificó su actuación, distribuyendo la asistencia a los centros educativos, objeto de la muestra.

A continuación, mantuvimos una reunión informativa con los directivos y los profesores de Música de los centros educativos y éstos cumplimentaron y aplicaron el Cuestionario a cada uno de los alumnos de la muestra, de forma individual. Se reunieron todos los datos para su estudio e interpretación.

\section{Análisis Estadístico}

Analizamos las frecuencias de los datos de cada uno de los Cuestionarios con objeto de analizar los items de mayor puntuación y hallamos los percentiles de las puntuaciones para seleccionar a todos los sujetos que obtenían un C. de 90 o superior. A continuación, se obtuvieron los porcentajes de alumnos de Infantil y de Primaria, de 5, 6, 7, 8, 9, 10 y 11 años, que obtenían dicho resultado. 
Tabla 1. Resultados del Cuestionario aplicado a los sujetos de 5 años, Educación Infantil Items: Media de las puntuaciones

\begin{tabular}{|c|c|c|c|c|c|c|c|}
\hline ITEMS & $\begin{array}{c}\text { Sujetos } \\
5 \text { años }\end{array}$ & $\begin{array}{c}\text { Sujetos } \\
6 \text { años }\end{array}$ & $\begin{array}{c}\text { Sujetos } \\
7 \text { años }\end{array}$ & $\begin{array}{c}\text { Sujetos } \\
8 \text { años }\end{array}$ & $\begin{array}{c}\text { Sujetos } \\
9 \text { años }\end{array}$ & $\begin{array}{c}\text { Sujetos } \\
10 \text { años }\end{array}$ & $\begin{array}{c}\text { Sujetos } \\
11 \text { años }\end{array}$ \\
\hline 1. & 1.47 & 1.44 & 1.17 & 1.52 & 1.43 & 1.6 & 1.7 \\
\hline 2. & 1.18 & 0.92 & 1.49 & 1.43 & 1.36 & 1.18 & 1.4 \\
\hline 3. & 0.5 & 0.4 & 0.64 & 0.68 & 0.34 & 1.19 & 0.85 \\
\hline 4. & 1.28 & 0.68 & 1.6 & 1.48 & 1.4 & 1.23 & 0.99 \\
\hline 5. & 1.53 & 1.04 & 0.95 & 1.39 & 1.2 & 1.3 & 1 \\
\hline 6. & 1.33 & 1.08 & 0.65 & 1.6 & 0.43 & 0.8 & 0.87 \\
\hline 7. & 1.29 & 0.6 & 0.97 & 1.14 & 0.67 & 0.9 & 1.08 \\
\hline 8. & 2 & 0.84 & 1.45 & 1.48 & 1.3 & 1.49 & 1.4 \\
\hline 9. & 1.29 & 1 & 1.33 & 1.77 & 1.5 & 1.68 & 1.8 \\
\hline 10. & 1.36 & 0.88 & 1.25 & 1,44 & 1.4 & 1.24 & 1.5 \\
\hline
\end{tabular}

El análisis de los datos refleja el resultado correspondiente a los items del cuestionario que mas destacan y obtienen una puntuación mayor en cada una de las edades, respecto a las habilidades propias del talento musical, de 5 años en Educación Infantil a 11 años en Educación Primaria. Los items que mas destacan se pueden observar en los gráficos siguientes.

Tabla 2 .Resultados del Cuestionario aplicado a los sujetos de 5 años, Educación Infantil. Sujetos con C igual a 90 o más.

$\begin{array}{lr}\text { Infantil } & 17,85 \% \\ \text { Primero } & 8 \% \\ \text { Segundo } & 5 \% \\ \text { Tercero } & 4,62 \% \\ \text { Cuarto } & 2,22 \% \\ \text { Quinto } & 0,93 \% \\ \text { Sexto } & 0 \%\end{array}$

Los datos en conjunto, según los resultados anteriores, indican que el talento musical decrece con la edad. El análisis estadístico muestra que los niños de Educación Infantil pueden tener un talento musical en un $17,85 \%$, pero estos resultados van descendiendo, hasta una cifra de un $0 \%$. 


\section{Gráfico 1. Resultados del cuestionario aplicado a los sujetos de 5 años, educación Infantil. Sujetos que obtienen un C igual a 90 o más.}

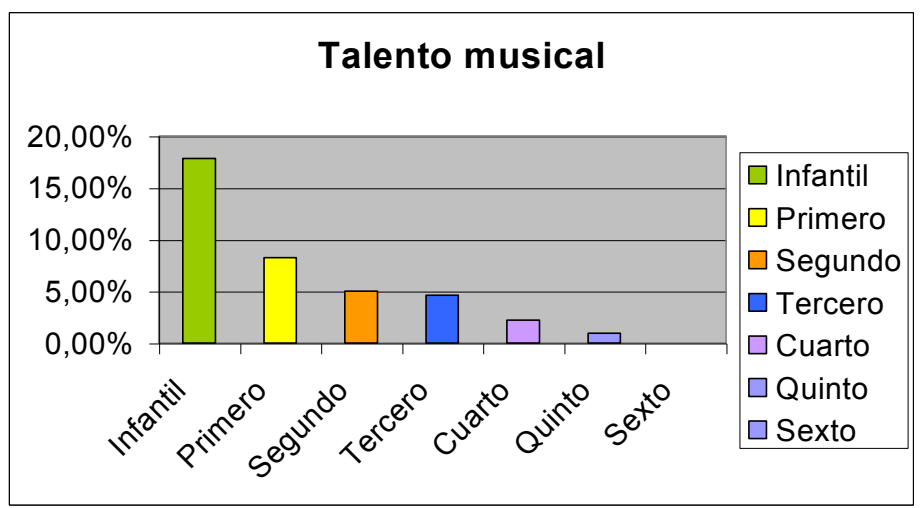

\section{Discusión}

El propósito de este estudio fue investigar sobre la identificación del talento musical desde diferencias de edad en los alumnos de 5 años de Educación Infantil, 6, 7, 8, 9, 10 y 11 años de Educación Primaria.

El análisis estadístico refleja que es posible la identificación del talento musical en Educación Infantil y que es superior a los cursos posteriores de Primaria. El análisis de destrezas del talento musical lleva a su conocimiento e identificación, tal y como se ha mostrado en estudios anteriores (véase H. Gardner, 1983; M. D.Prieto, 1997; A. Castelló y C. Batlle, 1998).

La aportación principal de este estudio a nuestro entender, estriba en mostrar la identificación de un tipo de talento, el musical, desde los 5 años en Educación Infantil y en los siguientes cursos de Primaria. Este trabajo lleva a concluir: a) Ya desde Educación Infantil es posible identificar el talento musical, como afirman diferentes autores. b) A partir de Primaria se observa una disminución de alumnos con talento musical. c)Para que el talento se desarrolle y permanezca en las sucesivos cursos de Primaria, se requiere su orientación y desarrollo. 


\section{Posibles limitaciones}

La primera limitación puede ser la diferencia de preparación de los profesores para aplicar el cuestionario de talento musical. Los profesores de música tienen mayor preparación que los maestros del aula de Educación Infantil y Primaria.

En cuánto a los estadísticos aplicados, se podría realizar un análisis comparativo respecto al género, ampliar la muestra a Secundaria y comparar las diferencias entre los diferentes grupos y aplicar una prueba de tendencia lineal. En este estudio no se llevó a cabo porque el objeto del trabajo se centró en la identificación del talento en las diferentes edades de Infantil y Primaria en el centro educativo.

En resumen, se puede afirmar que el talento musical se puede identificar desde edades tempranas, con la finalidad de intervenir en el desarrollo del talento para que permanezca durante los siguientes cursos de Primaria. Este estudio puede mostrar vías de identificación de los talentos en el contexto escolar para su orientación y desarrollo. 


\section{Referencias}

Armstrong, Th. (1994, 1999). Las Inteligencias Múltiples en el aula. Buenos Aires: Manantial. En Ferrándiz, C, Prieto M. D., García J.A., López O. Las inteligencias Múltiples: Un modelo de identificación de talentos específicos. (8) Revista Faísca de Altas Capacidades. Santiago de Compostela: Gríalibros.

Benito, Y. (1996). Inteligencia y algunos factores de la personalidad de los superdotados. Colección Psicología. Amarú: Salamanca.

Benito, Y. (1999). ¿Existen los superdotados? Praxis: Barcelona.

Birch, J. (1984). Is any identification procedure necessary? Gigted Child Quarterly, 28,4, 157-161.

Castelló, A. y Batlle, C. (1998). Aspectos teóricos e instrumentales en la identificación del alumnado superdotado y talentoso, propuesta de un protocolo. (6) Revista Faísca de Altas Capacidades. Santiago de Compostela: Gríalibros.

Gardner, H. (1983). Frames of mind: the theory of multiple intelligences. New York: Basic Books.

Gardner, H., Fieldman, D. H. y Krechevscky, M. (2000). El Proyecto Spectrum. Tomo I: Construir sobre las capacidades infantiles. Madrid: Ministerio de Educación, cultura y deporte y Morata.

Chen, J.-Q, Krechevscky, M., y Viens, J. Isberg, E. (2000). El Proyecto Spectrum. Tomo I: Construir sobre las capacidades infantiles. Madrid: Ministerio de Educación, cultura y deporte y Morata.

Gómez, A y Rodriguez, R. I.(1993). Talento. En L. Pérez (comp.). 10 palabras clave en superdotación. Estella, Navarra: Verbo Divino.

Martín Lobo, Ma P. (2004). Niños inteligentes. Guía para desarrollar sus talentos y altas capacidades. Colección Edu.com. Palabra: Madrid.

Mönks, F. J. (1994). Desarrollo emocional de los niños superdotados. En Benito Y., Intervención educativa en alumnos superdotados: 139-152. Amarú: Salamanca.

Prieto, M. D (1997). Evaluación y atención a la diversidad del superdotado. Aljibe: Málaga.

Renzulli, J. S. (1977). The enrichment triad model: A guide for developing defensible programs for the gifted and talented. Mansfield Center, CT: Creative Learning Press.

Renzulli, J. S. (1994). Schools for talent development: A practical plan for total school impovement. Msfield, CT: Creative Learning Press. 
Sánchez Manzano, E. (1999). Identificación de niños superdotaos en la Comunidad de Madrid. Ministerio de Educación y cultura: Madrid.

Sánchez Manzano, E. Dir. (2000). Alumnos superdotados: experiencias educativas en España. Ministerio de Educación y cultura: Madrid.

Sternberg R. J. (1995).La inteligencia excepcional. En R. Sternberg (ed.). Mas allá del CI. DDB: Bilbao.

Tourón, J., Peralta F., Reparaz Ch. (1998). La superdotación intelectual: Modelos, identificación y estrategias educativas. EUNSA: Pamplona. 
Anexo 1

CUESTIONARIO DE IDENTIFICACIÓN DEL TALENTO MUSICAL

COLEGIO:

Fecha.

NOMBRE Y APELLIDOS:

NIVEL ESCOLAR:

Edad:

\section{CUESTIONARIO SOBRE LA INTELIGENCIA MUSICAL}

Contestar en la casilla correspondiente en función de que le pase siempre, nunca o algunas veces.

\begin{tabular}{|c|c|c|}
\hline & 5 & No A.V. \\
\hline 1. Recuerdo con facilidad melodías y canciones. & & \\
\hline 2.Tengo buena voz para cantar. & & \\
\hline 3.Toco un instrumento musical o canta en un coro o en otro grupo. & & \\
\hline 4. Considero que tengo una manera rítmica de hablar y de moverme. & & \\
\hline 5. Tarareo melodías o canciones para mí mismo de forma inconsciente. & & \\
\hline 6.Golpeteo rítmicamente sobre la mesa o pupitre mientras trabajo. & & \\
\hline 7.Soy sensible a los ruidos ambientales. & & \\
\hline 8.Respondo favorablemente cuando suena una melodía musical. & & \\
\hline 9. Canto canciones aprendidas fuera del colegio. & & \\
\hline $\begin{array}{l}\text { 10. Tengo facilidad para identificar sonidos diferentes y percibir mati- } \\
\text { ces. }\end{array}$ & & \\
\hline
\end{tabular}

Datos de interés

- Observaciones del profesor de música.

- Asistencia a clases especializadas de música

- Asistencia a conciertos y tipo de música

- Participación en conciertos como cantante o tocando un instrumentos musical

- Se considera que tiene talento musical

- Su ambiente familiar es muy musical

- Otros aspectos que se consideren relevantes y no se pregunten: 